\title{
ESTUDIO SOBRE LA ADOPCIÓN DE VARIEDADES MEJORADAS DE FRIJOL EN LAS PRINCIPALES ZONAS PRODUCTORAS DE FRIJOL DE LA REGIÓN BRUNCA DE COSTA RICA ${ }^{1}$
}

\author{
Juan Carlos Hernández-Fonseca ${ }^{2}$, Flor Ivette Elizondo-Porras ${ }^{3}$
}

\begin{abstract}
RESUMEN
Estudio sobre la adopción de variedades mejoradas de frijol en las principales zonas productoras de frijol de la Región Brunca de Costa Rica. En Costa Rica, en el periodo 2000- 2004 se liberaron tres variedades de frijol grano rojo: Bribri (2000), Cabécar (2003) y Telire (2004). El proceso de generación y difusión de estas variedades, se efectuó bajo la estrategia de Fitomejoramiento Participativo (FP). En el estudio se entrevistaron 201 productores de cuatro localidades y 16 caseríos de la Región Brunca. El estudio consideró los años 2002, 2003 y 2004. En el periodo agrícola 2004 el $70 \%$ del área de siembra estuvo cubierta con las variedades Bribri (41,8\%), Cabécar (24,3\%) y Telire (3,3\%). El aumento del área de siembra con variedades mejoradas redujo el uso de variedades criollas, según los productores esto se debe a que las variedades criollas tienen un menor rendimiento y mayor susceptibilidad a las enfermedades. En el periodo 2004, el índice de aceptabilidad para la variedad Bribri fue de $51,8 \%$; este índice considera la adopción bajo los criterios del agricultor y el área sembrada con la variedad. Las localidades con niveles más altos de adopción, corresponden con las de mayor cantidad de agricultores organizados en Asociaciones de Productores; estas asociaciones son las que han participado más activamente en los procesos de FP. A partir de esta organización se derivan los factores que determinaron la adopción de la variedad Bribri, como es la participación en días de campo, días de evaluación de germoplasma y liberación de variedades de frijol. Se concluye de este estudio que existe adopción de la variedad Bribri y aceptación temprana de las variedades Cabécar y Telire.
\end{abstract}

\begin{abstract}
A study on the adoption of improved bean varieties in the main bean producing areas of the Brunca Region in Costa Rica. Three bean varieties were released in Costa Rica during the years 2000-2004 (Bribri, 2000, Cabecar, 2003 and Telire, 2004). The process of production and release of these varieties was conducted through the use of participative breeding (PB) methodology, with the objective of assuring their adoption by more farmers. Two hundred one producers were interviewed in four localities and 16 towns in the Brunca region during years 2002, 2003, and 2004. During the farming year 2004, $70 \%$ of the cropped land was planted with the varieties Bribri (41.8\%), Cabecar $(24.3 \%)$ and Telire $(3.3 \%)$. Planting with improved varieties significantly reduced the use of local lines, which were considered as less yielding and more susceptible to diseases by farmers. During year 2004, acceptability was $51.8 \%$, which includes adoption by farmers' criteria and area planted with this variety. The areas with higher adoption indexes correspond to those with a high percent of farmers organized in associations of producers, which have most actively participated in the PB process. Adoption of varieties was improved through farmer participation in field demonstrations, genotype evaluations, and release of bean cultivars. We conclude that the adoption of the Bribri variety has been accomplished and that there is early adoption of the varieties Cabecar and Telire.
\end{abstract}

Key words: Released varieties, planting area, Phaeolus vulgaris, red beans, black beans.

Palabras clave: Variedades liberadas, áreas de siembra, Phaseolus vulgaris, frijol rojo, frijol negro.

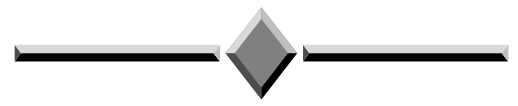

1 Recibido: 7 de junio, 2006. Aceptado: 5 de setiembre, 2006.

2 Instituto Nacional de Innovación y Transferencia en Tecnología Agropecuaria (INTA). Correo electrónico: j.hernandez@costarricense.cr

3 Dirección Nacional de Extensión Agropecuaria del Ministerio de Agricultura y Ganadería (MAG). Correo electrónico: fielizondo@ costarricense.cr 


\section{INTRODUCCIÓN}

La generación y transferencia de tecnología en frijol es un proceso dinámico, donde constantemente se pone a disposición de los productores diferentes recomendaciones cuyo objetivo es el de mejorar la productividad y producción del cultivo de frijol (Elizondo et al. 2004).

Durante el periodo 2000-2004 se liberaron en Costa Rica tres variedades de frijol de grano rojo (Bribri, 2000; Bribri, 2003; Telire, 2004), donde el proceso de generación y difusión de estas variedades, se realizó bajo la estrategia de fitomejoramiento participativo.

Los estudios de adopción son trabajos socioeconómicos que se efectúan tres o cuatro años después que se ha liberado una nueva opción tecnológica. En primera instancia permiten medir a nivel del productor, en el tiempo y espacio el uso de una determinada tecnología. Por otro lado, son instrumento de retroalimentación para orientar el trabajo de generación y transferencia, ya que se identifican factores agro-socioeconómicos que favorecen o limitan el uso de la propuesta tecnológica (CIMMYT 1993).

En Costa Rica, no existe información respecto a las tasas de uso de las variedades de frijol liberadas, en tal sentido resulta necesario hacer una investigación que permita contar con información relacionada con los agricultores y el área que actualmente se siembra con los nuevos materiales; y tal vez lo más importante, conocer los factores que expliquen la decisión del productor en cuanto a utilizar o no las nuevas variedades. El objetivo general de esta investigación es conocer las tasas de adopción de las nuevas variedades de frijol que se han liberado en Costa Rica e identificar los principales factores que las determinan.

\section{MATERIALES Y MÉTODOS}

\section{Zona de estudio}

Para realizar los estudios de adopción es necesario iniciar con un conocimiento exhaustivo de la zona de estudio, de la tecnología recomendada, de los productores y de las características básicas de las fincas productoras; esto permite conocer la población de estudio y posteriormente, definir la muestra a trabajar.

La investigación se llevó a cabo a principios del año 2005, en la Región Brunca, una de las principales regiones productoras de frijol del país. Se consideraron 16 caseríos ubicados en el distrito de Pejibaye del Cantón de Pérez Zeledón, y los distritos de Pilas y Chánguena del Cantón de Buenos Aires. Para la presentación de la información, se agruparon los caseríos en las localidades de Concepción de Pilas, Veracruz de Pejibaye, El Águila de Pejibaye y Chánguena.

\section{Tamaño de la muestra}

La información primaria se obtuvo del censo de casas de habitación del Ministerio de Salud y de los registros de asociados de las Asociaciones de Productores (ASOPRO).

Para determinar el número de agricultores a encuestar se aplicó la fórmula propuesta por Quintana (1989):

$$
n=\frac{-\left[\frac{Z \infty / \sqrt{p q}}{d}\right]}{1+\frac{1}{N}\left[\frac{Z \infty / 2 \sqrt{p q}}{d}\right]}
$$

Donde:

$\mathrm{n}=$ agricultores a encuestar

$Z \infty / 2=$ valor de probabilidad de la función normal, en este caso $Z=1,96$.

$\mathrm{d}=$ error de muestreo $5 \%$

$\mathrm{N}=$ número de productores de cada localidad

$\mathrm{p}=$ sí adopta

$\mathrm{q}=$ no adopta

La población muestreada fue de 201 agricultores ubicados al azar. La encuesta se diseñó a partir del aporte de Viana (2005) ${ }^{4}$.

4 VIANA, A. 2005. Estrategia para la aplicación de encuestas con pequeños agricultores en Centroamérica. IICA, Guatemala. Correo electrónico: aviana@iica.gt (Comunicación personal). 


\section{RESULTADOS}

\section{Caracterización de los productores de frijol}

\section{Edad}

El 58,8\% de los productores de frijol encuestados se encuentra entre los 31 y los 50 años, le sigue en orden de importancia los productores mayores de 50 años $(26,8 \%)$, y el grupo más pequeño es de los menores de 30 años $(16,4 \%)$. Con base en estas estadísticas, no se está dando el cambio generacional; ya que los jóvenes están optando por trabajar en actividades no agrícolas o por migrar a los Estados Unidos.

\section{Escolaridad}

Respecto al nivel educativo, la mayoría de los productores son alfabetas. Un $81,6 \%$ poseen educación primaria, de los cuales un 50,7\% de manera completa y algún grado de primaria aprobado un 30,9\%; solamente un $4,1 \%$ han recibido algún nivel de secundaria y un 13,9\% no tienen educación formal alguna.

\section{Organización}

En las zonas en estudio, el $57,7 \%$ de los productores dicen estar afiliados a alguna organización, en la mayoría de los casos, son asociaciones de productores legalmente constituidas. Los otros pertenecen a cooperativas, o a grupos locales como acueductos, patronato escolar, etc.

Dentro de estas organizaciones, han existido comités de investigación que son los responsables de coordinar las actividades de Fitomejoramiento Participativo, programando los días de campo y de evaluación de germoplasma, para divulgar a los agricultores y a la comunidad en general (Hernández y Araya 2004).

Con este proceso se ha logrado aumentar la participación del agricultor permitiendo una mayor y mejor divulgación de los resultados obtenidos; lo que facilitó la adopción de las nuevas variedades (Hernández y Araya 2004).
Los caseríos con mayor porcentaje de afiliados son Concepción con un 82,9 \%, Veracruz y Las Delicias con un $80,0 \%$. Por el contrario, los que tienen porcentajes más bajos de organización son Pilón y Cacique (Figura 1).

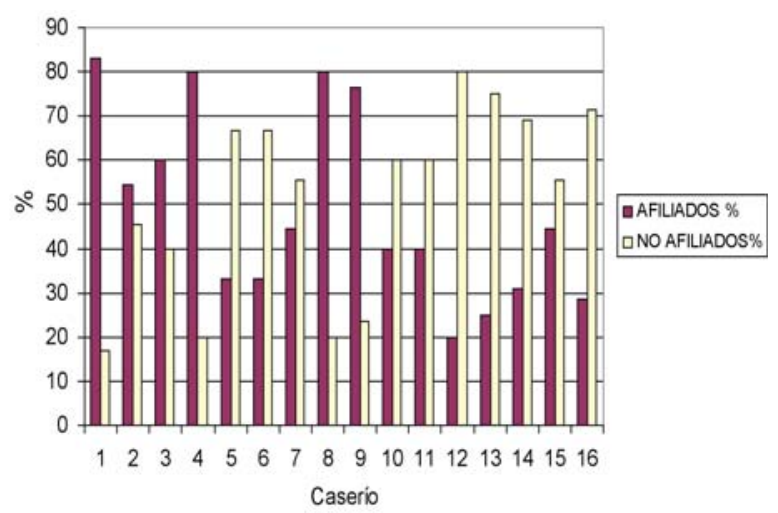

1: Concepción, 2: Pueblo Nuevo, 3: Guacimal, 4: Veracruz, 5: Bella Vista, 6: Guadalupe, 7: Santa Fe, 8: Las Delicias, 9: El Águila, 10: Chánguena, 11: Las Cruces, 12: Pilón, 13: Cacique, 14: La Bonita, 15: La Bonga, 16: Santa Lucía.

Figura 1. Distribución de los productores afiliados, por caserío. Región Brunca, Costa Rica. 2005.

En la Figura 2, se observa la distribución por localidades, en donde cerca del $70 \%$ de los agricultores de Chánguena no pertenecen a ninguna organización. Los agricultores que trabajan de forma aislada tiene menor probabilidad de adoptar variedades mejoradas; la participación en organizaciones locales destaca en

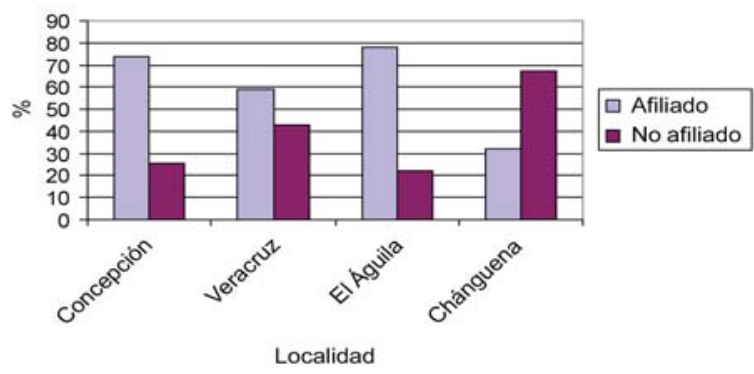

Figura 2. Distribución de los productores afiliados, por localidad. Región Brunca, Costa Rica. 2005.

AGRONOMÍA MESOAMERICANA 17(3): 357-367. 2006 
la decisión de usar variedades mejoradas (Sain et al. 1996; Viana et al. 1997b). Se logró determinar que la localidad y el pertenecer a una organización, efectivamente, son factores que ejercieron influencia sobre la adopción de variedades de frijol.

\section{Asistencia técnica}

En Concepción de Pilas, ninguno de los agricultores entrevistados recibe asistencia técnica en su finca por parte de las instituciones estatales localizadas en la zona. En las otras localidades, los que indican recibir asistencia son, en su mayoría, agricultores que participan o han participado en la conducción de ensayos de variedades de frijol. La asistencia técnica en fincas, no es un factor determinante en la adopción de variedades, ya que localidades con baja asistencia, poseen altos porcentajes de adopción. Los días de campo es la actividad de transferencia en las que más agricultores participan. La evaluación de ensayos de variedades de frijol, es un factor importante en localidades como Concepción, en donde el 66,7\% de los agricultores han participado. En la localidad de Chánguena, es donde menos actividades de transferencia se realizan, y es a su vez, la de menor adopción (Cuadro 1, Figura 3).

Según Viana et al. (1997a), los agricultores que reciben asistencia técnica aumentan la probabilidad de usar nuevas variedades.

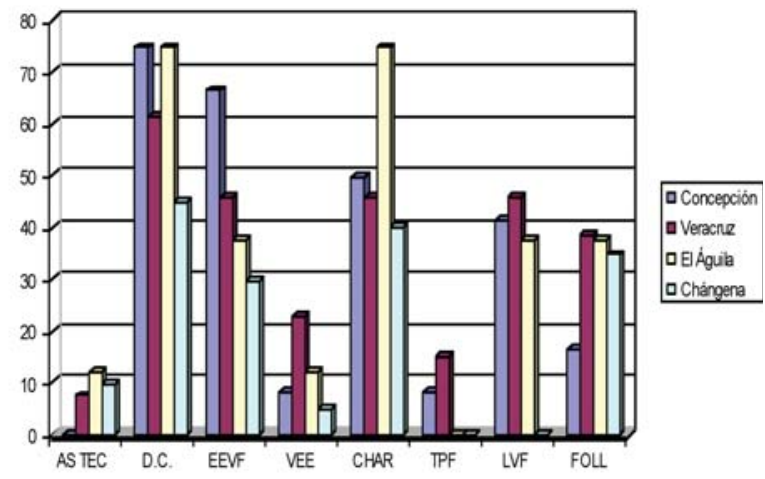

Figura 3. Participación de los agricultores en las diferentes actividades de transferencia de tecnología. Región Brunca, Costa Rica. 2005.

\section{USO DE VARIEDADES DE FRIJOL}

\section{Historial del uso de variedades de frijol}

Como se observa en el Cuadro 2, la variedad Bribri es conocida por el $75 \%$ de los agricultores entrevistados, valor similar al de la variedad Brunca (78\%). Este valor es importante ya que indica el rápido proceso de adopción de esta variedad, si se compara con la variedad Brunca que tiene más de 20 años de estar en el mercado. La intención de siembra para el año 2005, indica valores altos para las variedades Bribri y

Cuadro 1. Porcentaje de productores que han participado en diferentes actividades de transferencia. Región Brunca, Costa Rica. 2005.

\begin{tabular}{lcccc}
\hline \multirow{2}{*}{\multicolumn{1}{c}{ Actividad }} & \multicolumn{4}{c}{ Localidad } \\
\cline { 2 - 5 } & Concepción & Veracruz & El Águila & Chánguena \\
\hline \multirow{2}{*}{ Asistencia técnica en finca } & 0,0 & 7,7 & 12,5 & 10,0 \\
Días de campo & 75,0 & 61,5 & 75,0 & 45,0 \\
Evaluación ensayos de variedades & 66,7 & 46,2 & 37,5 & 30,0 \\
Visita a estación experimental & 8,3 & 23,1 & 12,5 & 5,0 \\
Charlas técnicas & 50,0 & 46,2 & 75,0 & 40,0 \\
Talleres PITTA frijol & 8,3 & 15,4 & 0,0 & 0,0 \\
Liberación de variedades & 41,7 & 46,2 & 37,5 & 0,0 \\
Información escrita sobre variedades & 16,7 & 38,5 & 37,5 & 35 \\
\% Adopción & 61,2 & 68,2 & 51,9 & 11,8 \\
\hline
\end{tabular}


Cuadro 2. Historial del uso de variedades mejoradas. Región Brunca, Costa Rica. 2005.

\begin{tabular}{|c|c|c|c|c|c|c|c|c|c|c|}
\hline \multirow[t]{2}{*}{ Variedad } & \multicolumn{2}{|c|}{$\begin{array}{c}\text { La } \\
\text { conoce }\end{array}$} & \multicolumn{2}{|c|}{$\begin{array}{l}\text { La ha } \\
\text { usado }\end{array}$} & \multicolumn{2}{|c|}{$\begin{array}{c}\text { Aún } \\
\text { la usa }\end{array}$} & \multicolumn{2}{|c|}{$\begin{array}{l}\text { Sembrará } \\
2005\end{array}$} & \multicolumn{2}{|c|}{$\begin{array}{c}\text { Dejó de } \\
\text { usarla }\end{array}$} \\
\hline & $\#$ & $\%$ & $\#$ & $\%$ & $\#$ & $\%$ & $\#$ & $\%$ & $\#$ & $\%$ \\
\hline Brunca & 157 & 78,11 & 157 & 78,11 & 52 & 25,87 & 44 & 21,89 & 105 & 52,24 \\
\hline Guaymi & 138 & 68,66 & 92 & 45,77 & 27 & 13,43 & 27 & 13,43 & 65 & 32,34 \\
\hline Chirripó & 122 & 60,70 & 60 & 29,85 & 2 & 1,00 & 3 & 1,49 & 58 & 28,86 \\
\hline Bribri & 152 & 75,62 & 121 & 60,20 & 93 & 46,27 & 95 & 47,26 & 28 & 13,93 \\
\hline Cabécar & 131 & 65,17 & 93 & 46,27 & 70 & 34,83 & 71 & 35,32 & 23 & 11,44 \\
\hline Telire & 85 & 42,29 & 36 & 17,91 & 26 & 12,94 & 38 & 18,91 & 10 & 4,98 \\
\hline
\end{tabular}

\#: Número de agricultores.

Cabécar. Hay una tendencia a disminuir las áreas de siembra con las variedades Guaymi y Brunca, debido al menor precio que se paga por el grano de color negro.

Los agricultores que dejaron de sembrar Bribri, argumentaron como principal razón, el no disponer de semilla.

\section{Variedades criollas más utilizadas}

El $73 \%$ de los entrevistados han usado recientemente una variedad criolla; de éstos, el 40\%, corresponde a la variedad Sacapobres. Le sigue en importancia Chimbolo con 26\%. Sin embargo, el área cubierta con Sacapobres no supera el $12 \%$. Esto nos indica que existe una diversidad, que los agricultores conservan, y que posiblemente sean las preferidas para su consumo. Algunas de estas variedades solo son reportadas por uno o dos agricultores, si en el corto plazo no se realiza una colecta de las variedades criollas, es posible que esta diversidad genética se pierda.

\section{ORIGEN DE LA SEMILLA DE FRIJOL}

Existen tres formas principales de adquirir la semilla de frijol: De la cosecha anterior, de un vecino y de la ASOPRO. Para las variedades Brunca y Guaymi, ambas de grano negro, la principal fuente es las siembras anteriores. Cerca del $30 \%$ de la semilla de las variedades Cabécar, Bribri, Telire y la criolla Sacapobres tienen como origen la Asociación de productores; es de resaltar el hecho que las ASOPROS, tienen amplia experiencia en la producción y comercialización de semilla con adecuados controles y han estado involucradas en evaluaciones sobre el impacto de la semilla de alta calidad (Hernández y Araya 2001).

En mayo de 2003, en las localidades de Veracruz y Concepción existían alrededor de cinco hectáreas con semilla de la variedad Cabécar, la cual fue obtenida por los agricultores de las parcelas de validación y se dedicaron a incrementarla al ver su potencial (Hernández y Araya 2003) (Figura 4).

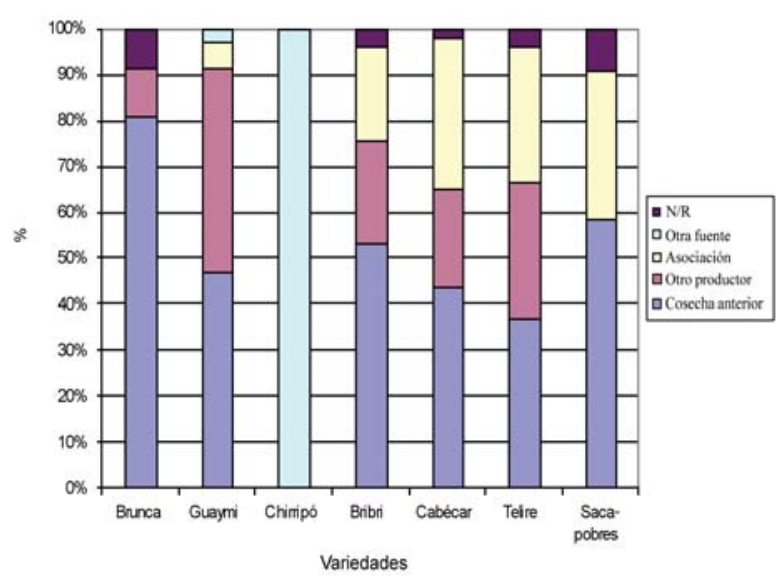

Figura 4. Origen de la semilla de las variedades de frijol. Región Brunca, Costa Rica. 2005. 


\section{ADOPCIÓN DE VARIEDADES MEJORADAS}

\section{Impacto de las variedades mejoradas}

El área de siembra con variedades mejoradas es del $80 \%$ (Figura 5); este aumento va en detrimento del área sembrada con la variedad Sacapobres y de otras variedades criollas de la zona. El motivo que los agricultores aducen para este cambio es el menor rendimiento de las variedades criollas con respecto a las mejoradas.

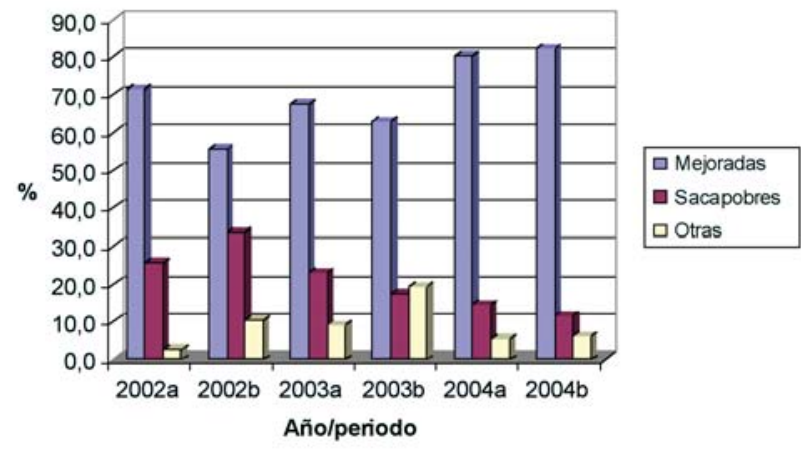

Figura 5. Porcentaje del área de siembra de variedades mejoradas en función de la variedad Sacapobres y otras variedades. Región Brunca, Costa Rica. 2005.

Se observa, cómo la mayoría de los productores sembró variedades mejoradas en el segundo ciclo del 2002. Un 34\% de ellos manifestó sembrar Sacapobres. Sin embargo, el área de siembra descendió del 22\% al $12 \%$ en dos años (Figura 5), mostrando tendencia manifiesta a desaparecer como variedad comercial.

En el Cuadro 3 y Figura 6, se observa como el área de siembra con las variedades Bribri, Cabécar y Telire, aumentó en un periodo de tres años. El mayor crecimiento fue para la variedad Cabécar; esto se debe a que existe expectativa y a que el grano posee mayor valor comercial por su color rojo claro. Telire es de más reciente liberación, pero también hay tendencia al aumento en el área sembrada. Por su parte Bribri, muestra en el tiempo estabilidad en el área de siembra. El primer ciclo de siembra es el de más importancia en la Región, por lo que el área de siembra a su vez es mayor con respecto al segundo ciclo.
Cuadro 3. Porcentaje del área de siembra de frijol con las variedades Bribri, Cabécar y Telire. Por año y ciclo de siembra. Región Brunca, Costa Rica. 2005.

\begin{tabular}{lccc}
\hline & \multicolumn{3}{c}{ Variedad } \\
\cline { 2 - 4 } Año/Ciclo & Bribri & Cabécar & Telire \\
\hline 2002 a & 44,0 & 5,1 & 0,0 \\
2002 b & 11,9 & 4,2 & 1,5 \\
2003 a & 37,6 & 11,0 & 1,4 \\
2003 b & 26,9 & 9,8 & 0,7 \\
2004 a & 41,8 & 24,3 & 3,3 \\
2004 b & 29,4 & 20,4 & 4,5 \\
\hline
\end{tabular}

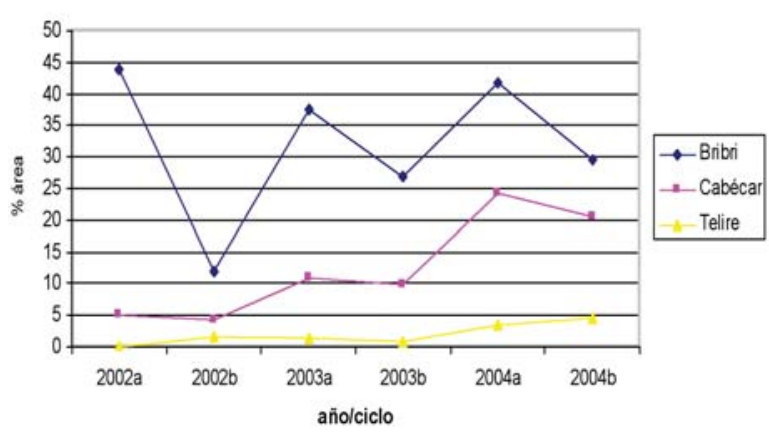

Figura 6. Porcentaje del área de siembra con las variedades Bribri, Cabécar y Telire. Por año y ciclo de siembra. Región Brunca, Costa Rica. 2005.

\section{ADOPCIÓN DE LA VARIEDAD BRIBRI}

\section{Impacto de la variedad Bribri}

La variedad Bribri fue liberada oficialmente en el año 2000. Sin embargo, el proceso de selección se inició desde el año 1995. Desde esta época los agricultores pertenecientes a las ASOPROS, participaban activamente en su evaluación y posterior producción de semilla. En el año 2001, en la primera época de siembra se estimaba que ya había sembrado con Bribri un $40 \%$ y un $25 \%$ del área, en Concepción y Veracruz respectivamente, esto indica la gran acogida de esta variedad (Hernández y Araya 2001). El porcentaje de agricultores que utilizan esta variedad se muestra en el Cuadro 4. En Guacimal, el porcentaje de uso de Bribri fue de un $100 \%$. En los caseríos de Chánguena, el uso 
Cuadro 4. Agricultores que utilizaron la variedad Bribri, por época y año, según caserío. Región Brunca, Costa Rica. 2005.

\begin{tabular}{llrrrrrr}
\hline \multirow{2}{*}{ Localidad } & \multicolumn{2}{c}{ Caserío } & \multicolumn{2}{c}{ Año 2002 } & \multicolumn{2}{c}{ Año 2003 } & \multicolumn{2}{c}{ Año 2004 } \\
\cline { 3 - 8 } & & 1era época & 2da época & 1era época & 2da época & 1era época 2da época \\
\hline \multirow{5}{*}{ Concepciónn } & 1. Concepción & 2,4 & 0,0 & 36,6 & 29,3 & 65,8 & 61,0 \\
& 2. Pueblo Nuevo & 0 & 0,0 & 0,0 & 0,0 & 9,1 & 27,3 \\
& 3. Guacimal & 0 & 0,0 & 20,0 & 20,0 & 100,0 & 80,0 \\
\hline \multirow{5}{*}{ Veracruz } & 4. Veracruz & 0 & 0,0 & 20,0 & 20,0 & 70,0 & 40,0 \\
& 5. Bella Vista & 0 & 0,0 & 0,0 & 0,0 & 33,3 & 0,0 \\
& 6. Guadalupe & 16,7 & 8,3 & 25,0 & 25,0 & 91,7 & 58,3 \\
& 7. Santa Fe & 11,1 & 11,1 & 22,2 & 11,1 & 44,4 & 44,4 \\
\hline \multirow{2}{*}{ Águila } & 8. Las Delicias & 0 & 0,0 & 50,0 & 30,0 & 60,0 & 60,0 \\
& 9. El Águila & 5,8 & 0,0 & 17,6 & 5,9 & 47,1 & 17,6 \\
& 10. Changuena & 0 & 0,0 & 0,0 & 0,0 & 0,0 & 6,7 \\
& 11. Las Cruces & 0 & 0,0 & 0,0 & 0,0 & 0,0 & 0,0 \\
& 12. Pilón & 0 & 0,0 & 0,0 & 0,0 & 0,0 & 6,7 \\
& 13. Cacique & 0 & 0,0 & 0,0 & 0,0 & 0,0 & 0,0 \\
& 15. La Bonita & 0 & 0,0 & 0,0 & 15,4 & 0,0 & 30,7 \\
& 16. La Bonga & 0 & 11,1 & 0,0 & 22,2 & 0,0 & 11,1 \\
& 17. Santa Lucía & 0 & 14,3 & 0,0 & 28,6 & 0,0 & 14,3 \\
\hline
\end{tabular}

es más limitado; sólo en La Bonita es de 30\%. La explicación se da por la menor participación de estos agricultores en los procesos de FP. Por localidad (Cuadro 5), se observa como en Concepción y Veracruz, en la época más importante de siembra (primer ciclo), más del $60 \%$ de los agricultores siembran esta variedad, comparativamente con Chánguena (sólo cultivan en segunda época) donde siembran cerca del $12 \%$ con Bribri. Esta se evidencia gráficamente en las Figuras 10 y 11.
En la Figura 7, se compara el área de siembra, la producción y los agricultores que utilizan la variedad Bribri. En Concepción, el porcentaje de gricultores es mayor que el porcentaje del área, por lo que se puede deducir que hay productores con áreas de siembra más pequeñas, comparativamente con El Águila. En Veracruz y Chánguena, el área de siembra es bastante similar. A nivel general (Figura 8), el porcentaje de agricultores en relación con el área de siembra de Bribri es muy similar.

Cuadro 5. Porcentaje de agricultores que utilizaron la variedad Bribri, por época y año, según localidad. Región Brunca, Costa Rica. 2005.

\begin{tabular}{lcrrrrr}
\hline \multirow{2}{*}{ Localidad } & \multicolumn{2}{c}{$\mathbf{2 0 0 2}$} & \multicolumn{2}{c}{ 2003 } & \multicolumn{2}{c}{ 2004 } \\
\cline { 2 - 7 } & Ciclo 1 & Ciclo 2 & Ciclo 1 & Ciclo 2 & Ciclo 1 & Ciclo 2 \\
\hline Concepción & 1,6 & 0,0 & 27,4 & 22,6 & 61,3 & 58,1 \\
Veracruz & 6,8 & 4,5 & 20,5 & 18,2 & 68,2 & 43,2 \\
El Águila & 3,7 & 0,0 & 29,6 & 14,8 & 51,9 & 33,3 \\
Changuena & 0,0 & 2,9 & 0,0 & 8,8 & 0,0 & 11,8 \\
\hline
\end{tabular}




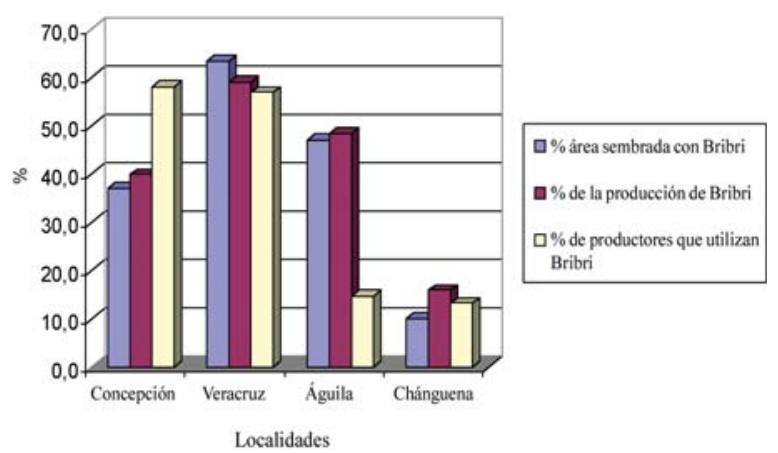

Figura 7. Porcentaje del área de siembra y de la producción con la variedad Bribri, por año y por ciclo de siembra. Región Brunca, Costa Rica. 2005.

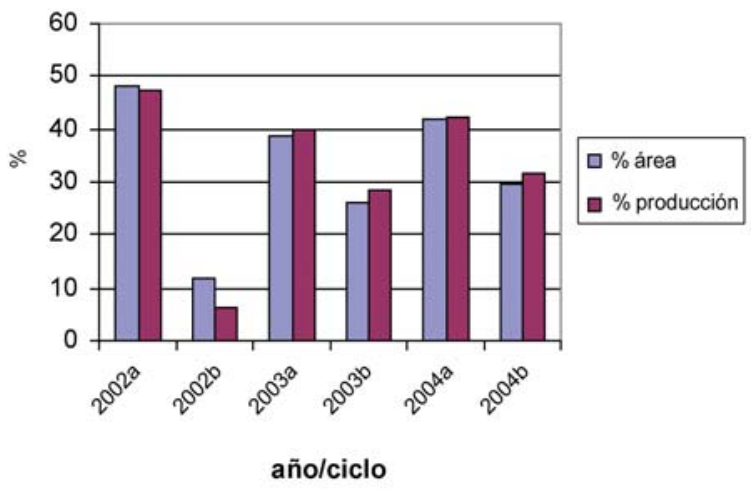

Figura 8. Porcentaje del área de siembra y de la producción con la variedad Bribri, por año y por ciclo de siembra. Región Brunca, Costa Rica. 2005.

El índice de aceptabilidad, es otro factor que denota la buena aceptación de la variedad Bribri; este índice considera la adopción desde el punto de vista del área sembrada con la variedad y del porcentaje de agricultores que la utilizan (PASOLAC 1999). Los datos del Cuadro 6 y la Figura 9, muestran como la aceptación aumenta, conforme pasan los años.

Según Hildebrand y Poey (1985), citado por PASOLAC (1999), el porcentaje de agricultores que aceptan la tecnología, debe de ser de al menos 50 y al mismo tiempo, el valor total del índice por lo menos de 25; en este caso, la tecnología tendría un buen potencial de aceptación. Valores obtenidos en este estudio se encuentran dentro de estos rangos,

ISSN: 1021-7444
Cuadro 6. Índice de aceptabilidad de la variedad Bribri. Región Brunca, Costa Rica. 2005.

\begin{tabular}{ccccc}
\hline Año & Ciclo & $\begin{array}{c}\text { \% de } \\
\text { agricultores }^{\mathbf{a}}\end{array}$ & $\begin{array}{c}\text { \% del } \\
\text { área }^{\mathbf{b}}\end{array}$ & I.A. \\
\hline & 1 & 22,73 & 48,3 & 30,9 \\
2002 & 2 & 10,81 & 11,9 & 11,3 \\
\hline & 1 & 53,97 & 38,5 & 44,9 \\
2003 & 2 & 35,16 & 26,1 & 30,0 \\
\hline & 1 & 66,13 & 42,6 & 51,8 \\
2004 & 2 & 41,14 & 29,4 & 34,3 \\
\hline
\end{tabular}

$\mathrm{IA}=2 /(1 / \mathrm{a})+(2 / \mathrm{b}) \times 100$

IA: Índice de aceptabilidad.

$\mathrm{a}=$ La proporción de adopción bajo el criterio productor.

$\mathrm{b}=$ La proporción de adopción bajo el criterio área.

principalmente para la primera época de siembra de cada uno de los años en estudio.

\section{Ventajas de la variedad Bribri}

La principal ventaja que resaltan los agricultores entrevistados de la variedad Bribri es su buen rendimiento (88\%), le sigue la resistencia a las enfermedades y el buen porte de la planta. Estas características tienen estrecha relación entre sí, ya que el porte de Bribri, le brinda un menor contacto de las vainas con el suelo (posible contacto con patógenos), y un ambiente de mayor circulación del aire (Figura 10).

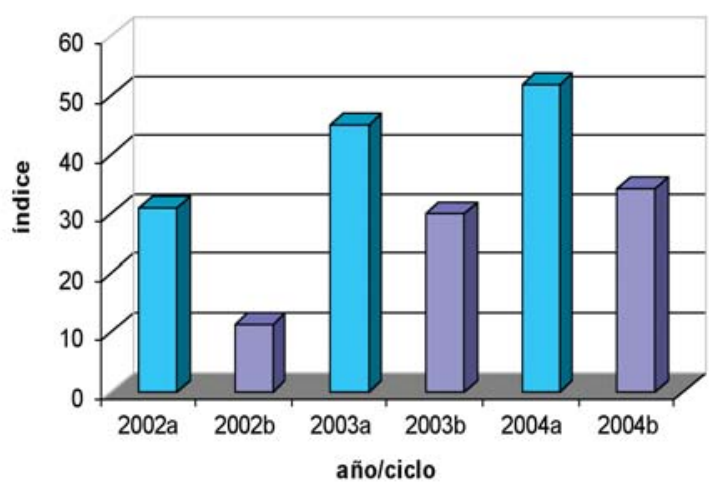

Figura 9. Índice de aceptación de la variedad Bribri. Región Brunca, Costa Rica. 2005. 


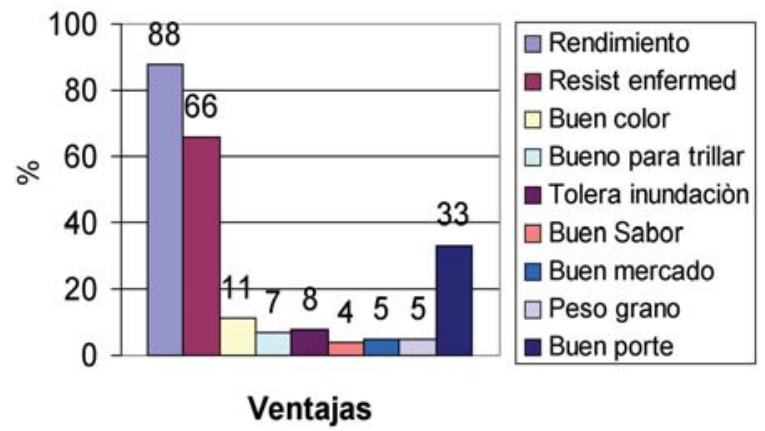

Figura 10. Principales ventajas de la variedad Bribri. Región Brunca, Costa Rica. 2005.

\section{Desventajas de la variedad Bribri}

Los agricultores indican que la variedad Bribri, es susceptible al amachamiento, un $25 \%$ de ellos así lo manifiestan. El $12 \%$ mencionan que el color de la variedad es malo (Figura 11); esta característica va a ser determinante en el tiempo afectando la permanencia de esta variedad en el mercado, ya que la demanda actual es por variedades de grano rojo claro.

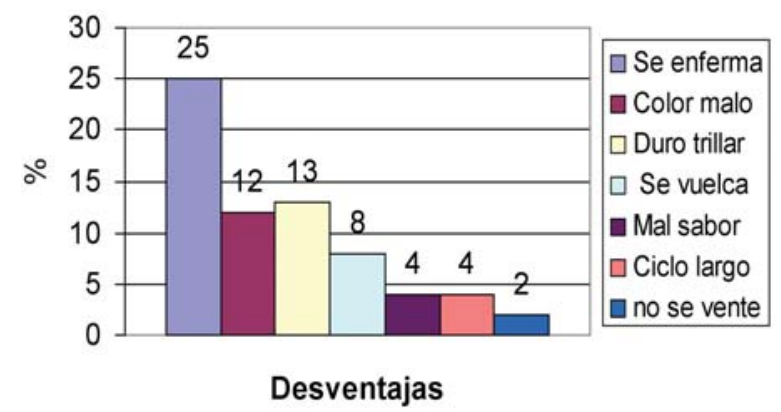

Figura 11. Principales desventajas de la variedad Bribri. Región Brunca, Costa Rica. 2005.

\section{PRINCIPALES CARACTERÍSTICAS DE LAS VARIEDADES}

Las principales características que buscan los agricultores en una nueva variedad de grano rojo son: Rendimiento, resistencia a enfermedades, y el porte de la planta. Características mencionadas por Mazariego y Argueta (2004), en donde los agricultores buscan además, la consistencia del caldo y el color de grano.

\section{EL FITOMEJORAMIENTO PARTICIPATIVO Y LA ADOPCIÓN DE VARIEDADES}

En el fitomejoramiento participativo, la transferencia de material genético siempre se realiza de manera participativa; es decir, el agricultor participa activamente en todas las fases. Esta metodología de transferencia permite que el agricultor conozca y se convenza de la nueva tecnología por anticipado, de manera que cuando esta es liberada, ya muchos de los productores la conocen y la adoptan. La participación de los agricultores fue indispensable, para una selección apropiada del material (basada en su experiencia de manejo agronómico) así se han logrado implementar procesos de adopción más rápido, al difundir tecnología que ya es conocida y apropiada para las condiciones de los productores, dado que el producto final contiene los criterios del productor para aceptar o rechazar una tecnología (Araya et al. 2002; Elizondo, et al. 2004). Por ejemplo, al liberar la variedad Cabécar en el 2003, gran cantidad de agricultores conocía el material, el cual había sido sembrado en sus parcelas. Lo mismo sucedió con la variedad Bribri que en el año de su liberación (2000), ya había alrededor de 60 hectáreas sembradas (Hernández, y Araya 2001; Hernández y Araya 2003).

Los agricultores participantes en la validación de la línea EAP 9510-77, se mostraron satisfechos con el comportamiento agronómico, por lo que solicitaron al PITTA frijol su liberación, en respuesta fue liberada oficialmente como Cabécar (Hernández y Araya 2004).

El estudio ha permitido determinar que el FP es más efectivo, por cuando los agricultores participan en la estrategia misma de transferencia, al ser parte de la organización de los mismos. Además, se genera más confianza entre los actores (productores e investigadores) en los resultados obtenidos (Elizondo et al. 2004).

\section{IMPACTO DE LOS ESTUDIOS DE ADOPCIÓN}

Todas la instituciones que participen en la generación y transferencia de tecnología agrícola, deben tener capacidad de realizar estudios que documenten el grado de adopción y ayuden a explicar lo que 
motiva las decisiones de los agricultores (CIMMYT 1993).

El estudio de adopción permite obtener información de los agricultores, que será útil para afinar la generación de tecnología, dado que los productores al seleccionar una variedad consideran los requerimientos del mercado y las necesidades del usuario del producto final (CIMMYT 1993; Elizondo et al. 2004).

Es posible a través de un estudio de adopción de variedades, definir cuál estrategia de transferencia es la más adecuada para lograr la adopción de las variedades, evidentemente las actividades en donde el agricultor pueda observar y comparar el comportamiento de un grupo de materiales, y tomar la decisión de cual se adapta a sus condiciones, es la mejor estrategia de transferencia.

Estos estudios permiten documentar los efectos de un proyecto de FP. La evaluación de las repercusiones de la investigación y la extensión, y medir los beneficios que se pueden derivar de la aplicación de los resultados de la investigación.

Un aspecto importante es el cambio de actitud en los investigadores en cuanto al papel del productor en este proceso, por cuanto valoran sus conocimientos y sus necesidades al incorporarlos en los procesos de evaluación (Elizondo et al. 2004). Además, permite recopilar el conocimiento práctico que tienen los agricultores sobre el medio, comprender los criterios que utilizan para evaluar una variedad, que por lo general son desconocidos por el investigador. La interacción entre ambos durante la investigación, proporciona la primera indicación de si es o no es aceptable una variedad (CIMMYT 1993).

\section{CONCLUSIONES}

Hay diversidad de variedades criollas en la zona donde se evaluó la adopción de variedades mejoradas. Sin embargo, aunque un porcentaje muy alto de los productores las utilizan, es común que algunas de éstas solo estén en manos de uno o dos productores y en áreas pequeñas. En el corto o mediano plazo, podría implicar erosión genética y una pérdida irreversible de esta diversidad, se debería hacer esfuerzo para colectar y posteriormente caracterizar estas variedades.
En la zona de estudio hay un alto uso de variedades mejoradas (80\%); sin embargo, su utilización implica un desplazamiento de las variedades criollas.

Hay una tendencia a la disminución de las áreas de siembra de las variedades de grano negro; la principal razón es el menor precio que se paga por el grano de este color.

Una mayor adopción se puede dar cuando los agricultores están organizados en asociaciones de productores, ya que estos grupos a su vez participan activamente en al trabajo en fitomejoramiento participativo, lo que permite a los afiliados tener contacto con las variedades, mucho antes de ser oficialmente liberadas.

Las principales características que los agricultores indicaron en una nueva variedad mejorada de grano rojo fueron: el rendimiento, la resistencia a enfermedades y el porte de la planta.

De este estudio se concluye que existe adopción de la variedad Bribri y aceptación temprana de las variedades Cabécar y Telire.

\section{AGRADECIMIENTOS}

La realización de este estudio fue auspiciada por, el Bean/Cowpea CRPS (Donación USAID GDG-G-0002-00012-0), a través de la Escuela Agrícola Panamericana, Zamorano, Honduras. Se contó con la colaboración de la M.Sc. Beatriz Sandoval del Instituto Nacional de Innovación y Transferencia en Tecnología Agropecuaria (INTA) en el diseño de la muestra; y del Dr. Olman Quirós Madrigal del Ministerio de Agricultura y Ganadería, en el procesamiento y análisis de los datos.

Un especial agradecimiento a las asociaciones de productores de Veracruz de Pejibaye, Concepción de Pilas, El Águila de Pejibaye y Chánguena de Buenos Aires, así como a sus asociados y productores de frijol en general, los cuales estuvieron anuentes en todo momento a brindar la información requerida.

A los funcionarios del Ministerio de Agricultura y Ganadería, y del Consejo Nacional de Producción, ubicados en Pejibaye de Pérez Zeledón, Buenos Aires y Potrero Grande de Buenos Aires, por su colaboración en la recolección de la información. 
Al Dr. Juan Carlos Rosas de la Escuela Agrícola Panamericana, El Zamorano. Rica.

A los demás miembros del PITTA Frijol de Costa

\section{LITERATURA CITADA}

ARAYA, R.; HERNÁNDEZ, J.C.; ELIZONDO, F.I. 2002. El fitomejoramiento participativo en la selección de líneas promisorias de frijol para grupos organizados de pequeños productores de la Región Brunca, Costa Rica. I. In: VI Taller anual de resultados de investigación y transferencia de tecnología. Agosto 2002. San José, Costa Rica. p. 35-40.

CIMMYT. 1993. La adopción de tecnologías agrícolas: Guía para el diseño de encuestas. Programa de Economía. México, D.F. 88 p.

ELIZONDO, F.I.; HERNÁNDEZ, J.C.; ARAYA, R. 2004. La evaluación participativa en los procesos de fitomejoramiento del frijol In: VIII Reunión anual del sector frijolero de Costa Rica. Agosto 2003. San Carlos, Costa Rica. p. 25-28.

HERNÁNDEZ, J.C.; ARAYA, R. 2001. Fitomejoramiento participativo en la Región Brunca de Costa Rica. In: $\mathrm{V}$ Taller anual de resultados de investigación y transferencia de tecnología. Agosto 2001. Alajuela, Costa Rica. p. 97-102.

HERNÁNDEZ, J.C.; ARAYA, R. 2003. Cabécar, variedad de frijol de grano rojo para costa Rica. In: VII Reunión anual del sector frijolero de Costa Rica. Setiembre 2003. Heredia, Costa Rica. p. 21-28.
HERNÁNDEZ, J.C.; ARAYA, R. 2004. Logros en la implementación del Fitomejoramiento Participativo (FP) en frijol en Costa Rica.l In: VIII Reunión anual del sector frijolero de Costa Rica. Agosto 2003. San Carlos, Costa Rica. p. 13-24.

MAZARIEGO, M.; ARGUETA, O. 2004. Estudio de aceptación de la variedad de frijol "CENTA San Andrés". Ministerio de Agricultura y Ganadería, Centro Nacional de Tecnología Agropecuaria y Forestal (CENTA). El Salvador. 26 p.

PASOLAC, 1999. Índice de introducción de una herramienta sencilla de seguimiento a la transferencia - con dos ejemplos. Documento \# 224, Serie Técnica. 9/99. Managua, Nicaragua. 47 p.

QUINTANA, C. 1989. Elementos de inferencia estadística. Editorial Universidad de Costa Rica. San José, Costa Rica. 73 p.

SAIN, G.; HERRERA F.; MARTINEZ, J.1996. Adopción y uso de semilla mejorada de maíz entre pequeños agricultores de Guatemala. ICTA-PRM-CIMMYT. San José, Costa Rica. 52 p.

VIANA, A.; RODRIGUEZ, F.; ESCOTO, D. 1997a. Adopción de la variedad Dorado en la región centro-oriental de Honduras. In: Seminario taller: Métodos para medir la adopción de nuevas tecnologías, resumen de experiencias y lecciones para el futuro. CIMMYT, PRM, IICA, PROFRIJOL, PASOLAC. San José, Costa Rica. 1997.

VIANA, A.; MUNGUÍA, R.; GA, L. 1997b. Adopción de las variedades Estelí 90, en las Segovias, Nicaragua. In: Seminario taller: Métodos para medir la adopción de nuevas tecnologías, resumen de experiencias y lecciones para el futuro. CIMMYT, PRM, IICA, PROFRIJOL, PASOLAC. San José, Costa Rica. 1997. 